\title{
PROMOCIÓN Y PREVENCIÓN DEL DETERIORO COGNITIVO Y DEMENCIAS EN PERSONAS MAYORES
}

\section{Promotion AND PREVENTION OF COGNITIVE DETERIORATION AND DEMENTIA IN OLDER PEOPLE}

\author{
Lorena Soledad Ayala San Martín
}

Enfermera Magister en Gestión en Atención Primaria de Salud, Académica Universidad Católica del Maule, Calca, Chile layala@ucm.cl tesis para acceder al grado de magister, Facultad de Enfermería, Universidad Andrés Bello. 


\section{Resumen}

El envejecimiento poblacional a nivel mundial ha llevado a un aumento de la prevalencia de enfermedades neurodegenerativas, presentándose como un desafío para los equipos de salud. El objetivo de este trabajo es analizar evidencia científica relacionada con la prevención del deterioro cognitivo y demencias, a nivel nacional e internacional en personas mayores.

Método: Revisión de evidencia científica disponible. Resultados: Se seleccionaron 15 artículos, de los cuales 1 es nacional, donde se realiza una reseña de los factores protectores y de riesgo para la presentación de demencias en personas mayores. Los otros 14 estudios son internacionales, 7 son estudios experimentales donde se aplica a los sujetos de estudio un programa de actividades en la comunidad que resultaron positivas para el desempeño cognitivo. Los otros 7 estudios son observacionales donde se analizan factores protectores para la prevención de la demencia.

Conclusión: Los factores de riesgo para la presentación de deterioro cognitivo leve y demencias son similares a otras enfermedades crónicas no transmisibles, el trabajo sobre factores protectores, permitiría mejorar el rendimiento cognitivo y retardar el avance de las demencias en etapas iniciales. Se requieren estudios locales y a largo plazo para observar la realidad nacional.

Palabras Claves: Disfunción Cognitiva; Anciano; Prevención; Demencias; Cognitive Dysfunction; Aged; Prevention; Dementias; Disfunção Cognitiva, Idoso, Prevenção \& Controle, Demência. 


\section{Abstract}

Population aging worldwide has led to an increase in the prevalence of neurodegenerative diseases, presenting itself as a challenge for health teams. The objective of this work is to analyze scientific evidence related to the prevention of cognitive impairment and dementias, nationally and internationally, in older people.

Method: Review of available scientific evidence. Results: 15 articles were selected, of those 1 is national, where made review of the protective and risk factors for the presentation of dementias in older people is made. The ot ${ }^{-}$ her 14 studies are international, 7 are experimental studies where a program of activities in the community that were positive for cognitive performance is applied to the study subjects. The other 7 studies are observational where protective factors for the prevention of dementia are analyzed.

Conclusion: The risk factors for the presentation of mild cognitive impairment and dementias are similar to other chronic non-communicable diseases, the work on protective factors, would improve cognitive performance and delay the advance of dementias in the initial stages. Long-term and local studies are required to observe the national reality.
Keywords: Cognitive Dysfunction; aged; Prevention; Dementias; 


\section{INTRODUCCIÓN}

La población a nivel mundial está envejeciendo, las mejoras en la atención de la salud del último siglo han contribuido a que las personas tengan vidas más largas y saludables. ${ }^{1}$

Chile cuenta con dos y medio millones de personas mayores representando el $12,9 \%$ de la población total, situación que irá en aumento, se estima que en el 2050 esta proporción aumentará a un $30 \%$ de la población total. ${ }^{2}$

El aumento de la esperanza de vida en los últimos años, con el consiguiente envejecimiento de la población, ha supuesto un aumento de la prevalencia de las enfermedades neurodegenerativas que cursan con deterioro cognitivo. ${ }^{2}$

El deterioro cognitivo leve es un estado en el cual las personas presentan problemas de memoria mayor a la esperada durante el envejecimiento, el cual no afecta al desarrollo de actividades de la vida diaria, ${ }^{3}$ no así, es el caso de las demencias, síndrome mayormente de naturaleza crónica o progresiva, causado por una variedad de enfermedades cerebrales que afectan la memoria, el pensamiento, el comportamiento y la habilidad de realizar actividades de la vida diaria. $^{3}$

Las terapias de estimulación cognitiva han mostrado generalmente resultados positivos en los estudios y metaa- nálisis realizados, con una mejoría en la función cognitiva, y en otros factores, como calidad de vida e interacción social. 4

En el presente estudio se pretende realizar una revisión de la evidencia científica de las distintas programas de intervención que se hayan investigado a nivel nacional e internacional, de esta forma es posible conocer la efectividad de estos en la población de estudio, lo cual podría aportar un precedente para el desarrollo de futuras estrategias que vayan en pro de la prevención del deterioro cognitivo o demencias en etapa inicial, a nivel local.

\section{Metodología}

Esta revisión fue realizada en base a la búsqueda de evidencia científica disponible relacionada con la prevención del deterioro cognitivo y demencias en personas mayores, se utilizaron distintos motores de búsqueda y palabras claves de investigación reconocidos en MesH y Bireme, enlazados por el booleano "and". Posteriormente se realizó una revisión exhaustiva de esta selec $^{-}$ cionándola con los criterios de inclusión que se describirán más adelante.

Se realizó una búsqueda bibliográfica en las bases de datos: PubMed, Dialnet, SCIELO (Scientific Electronic Library Online) y Epistemónikos. 
Los descriptores específicos reconocidos por MeSH y bireme para filtrar la búsqueda serán: "Cognitive Dysfunction", "Aged", "prevention", "dementia", "prevención", "demencias".

El operador booleano utilizado en la búsqueda es: "and".

Como dentro de los criterios de búsqueda se encuentraba "Aged", esto dirige a que los principales sujetos de estudios presentan esta característica, ayudando a que los conceptos "cognitive dysfunction", "prevention", "dementia" sea complementario, todos unidos por el booleano "and".

Para realizar la búsqueda de evidencia se utilizaron las siguientes bases de datos científicas:

- Pubmed.

- Dialnet.

- Scielo

- Epistemónikos.

\section{Filtros metodológicos para la}

\section{búsqueda}

Una vez realizada la búsqueda en los motores señalados anteriormente, se realizó filtro en base a los criterios de inclusión, descartando a los estudios que no se encuentran dentro de estos, textos no disponibles, sujetos de estudio de otro grupo etario o que se encontraban publicados en más de una base bibliográfica revisada.

\section{Criterios de inclusión y exclu-} sión para la búsqueda

Los criterios de inclusión y exclusión en la ruta de búsqueda fueron: grupo etario descrito como personas mayores de 60 años, artículos que se encontraron en inglés y español, disponibles y evidencia de los 5 últimos años (enero del 2012 a junio del 2017), estudios con metodología cuantitativa: ensayos clínicos aleatorios, descriptivos, observacionales, longitudinales, interpretativos y revisión narrativa. Se excluirán los estudios cualitativos, también aquellos que arrojó la búsqueda, pero que no se encontraron como texto disponible, que no fueron sujetos de estudios personas mayores y en una sola versión de su publicación.

\section{REsultados}

Búsqueda y selección de evi dencia

Pubmed arrojó como resultado 32 tipos de documentos disponibles de los cuales cumplieron con los criterios de selección solo 2. Con el segundo buscador Dialnet la búsqueda arrojó 278 documentos, que posterior a la revisión fueron descartados los que no cumplían con 
los criterios de inclusión, seleccionando finalmente 9 documentos. SCIELO arrojó 8 documentos de los cuales sólo $1 \mathrm{cum}^{-}$ plió con los criterios de inclusión.

Y en el último buscador, Epistemónikos, la búsqueda arrojó un total de 259 estudios, de los cuales 21 cumplían con los criterios de inclusión.

A pesar de que el número de estudios que cumplieron con los criterios de inclusión en cada buscador, existe un número de estudios que se repiten en el resultado de búsqueda, por lo tanto se seleccionaron en una versión de publicación.

Artículos según lugar de intervención

Artículos Nacionales:

Solo uno de los artículos encontrados es de origen nacional, el dónde se realizó una reseña de la investigación disponible, respecto a factores de riesgos para desarrollar deterioro cognitivo leve y probable tránsito hacia la demencia, asociándolo a otras afecciones como la depresión, Parkinson y otras de tipo neurodegenerativas. Destaca que frente a lo observado se hace importante trabajar de manera preventiva, a través de programas dirigidos a las personas mayores.

Artículos internacionales:

El resto de los artículos son estudios internacionales, donde se realizó algún tipo de intervención de tipo no farmacológica a personas mayores, tanto en la comunidad como en unidades de larga estadía, el fin de las intervenciones es prevenir el deterioro cognitivo y demencias en personas con envejecimiento normal, o preservar la función cognitiva en sujetos mayores que se encontraban en etapas iniciales de deterioro cognitivo o demencia, específicamente enfermedad de Alzheimer.

Intervención en prevención de DCL y demencias

El análisis mayormente realizado en esta Revisión sistemática es de intervenciones de prevención primaria y secundaria en personas mayores con envejecimiento normal, deterioro cognitivo leve y etapa inicial de las demencias. Los estudios analizados utilizaron distintas metodologías, y fueron realizadas en distintos países del mundo, en personas mayores con y sin institucionalización.

De las investigaciones donde los sujetos de estudios no presentaban institucionalización se encontraron los siguientes:

-"Mejoras cognitivas y perceptivomotrices en personas mayores participantes en un programa de estimulación integral cognitivo motriz", PontevedraEspaña. 5 
- "Efectos de la terapia no farmacológica en el envejecimiento normal y el deterioro cognitivo: consideraciones sobre los objetivos terapéuticos", MadridEspaña. 6

- "El hábito de lectura como factor protector de deterioro cognitivo", Valencia- España.7

- "Total daily physical activity and the risk of $A D$ and cognitive decline in older adults", Chicago USA. 8

- "Mental and Physical Activities Delay Cognitive Decline in Older Persons with Dementia”, Hong Kong, China. ${ }^{9}$

- "Effects of Intervention Using a Community-Based Walking Program for Prevention of Mental Decline: A Randomized Controlled Trial", Takasaki, Japó$\mathrm{n}^{10}$

- "Late life leisure Activities And Risk of Cognitive Decline", Sichuan y Shandong, China. ${ }^{11}$

- "1-Year Randomized Controlled Trial Comparing Mind Body Exercise (Tai Chi) With Stretching and Toning Exercise on Cognitive Function in Older Chinese Adults at Risk of Cognitive Decline", Hong Kong, China. 12

- "Social isolation and loneliness: relationships with cognitive function during 4 years of follow-up in the English Longitudinal Study of Ageing", Londres, Inglaterra. ${ }^{13}$
- "Physical activity improves verbal and spatial memory in older adults with probable mild cognitive impairment: a 6-month randomized", Vanocuver, Canadá. ${ }^{14}$

- "Feelings of loneliness, but not social isolation, predict dementia onset: results from the Amsterdam Study of The Elderly (Amstell)", Amsterdam- Holanda ${ }^{15}$

- "A randomized controlled trial of multicomponent exercise in older adults with mild cognitive impairment", ObuJapón. 16

De las investigaciones donde los sujetos de estudios se encontraban institucionalizados se encuentran los siguientes:

- "Estudio piloto de un nuevo método de estimulación aritmética empleando el ábaco en ancianos sanos y con trastorno cognitivo". Servicio de Neurología del Hospital Clínico San Carlos de Madrid y la Sección de Neurología del Hospital Universitario Infanta Cristina de Parla, Madrid. Madrid España. ${ }^{17}$

- "Factores predictores de deterioro cognitivo en población mayor de 64 años institucionalizada y no institucionalizada”. Huelva- España.18

De los estudios analizados un total de 12 evaluaron el nivel cognitivo de los sujetos de estudios, dentro de las 
herramientas utilizadas se reconocieron en 4 el minimental state (MMSE), 2 el mini examen cognoscitivo de Lobo, en 1 estudio el geriatric mental state (GMS), 1 estudio aplicó un cuestionario de 25 pre- $^{-}$ guntas de autocompletación concernientes al deterioro mental, en 1 estudio se aplicó el instrumento "Community Screening Instrument for Dementia" (CSID), 1 estudio ocupó la escala "Clinica dementia Rating" (CDR) de Hughes, 1 estudio evaluó afectividad a través de la ansiedad y la depresión y la autopercepción de la calidad de vida, del resto de los estudios analizados utilizaron distintos exámenes de evaluación cognoscitivas. En 1 estudio se midió la memoria verbal y aprendizaje mediante el test de aprendizaje auditivo y verbal de Rey (RAVLT) y la memoria espacial utilizando un examen computarizado. En 1 estudio se mide la capacidad de realizar actividades de la vida diaria a través de la escala de Barthel, función motora a través del "time up and go", capacidad de actividad física a través de actigrafìa, la salud general a través del goldberg, el hábito de lectura (frecuente, ocasional o no lector) según criterio de la federación de gremios y editores de España (FGEE).
Intervenciones a nivel interna cional

En la búsqueda de evidencia sobre intervenciones de prevención de deterioro cognitivo y/o demencias se encontraron un gran número de estudios en países como: China, Japón, España, EEUU, Canadá y Cuba, no así en países de Latinoamérica.

Dentro de la evidencia seleccionada se observaron distintos programas de intervención para la prevención o tratamiento de deterioro cognitivo o demencias en etapa inicial. De las intervenciones destacan mayormente programas de actividad física, estimulación cognitiva y participación social, también se relacionaron con los años de educación formal y el hábito de lectura presente en los últimos 5 años. En todas las intervenciones estudiadas se observó un mejor rendimiento cognitivo posterior a las intervenciones.

\section{Discusión}

En la presente revisión se realizó un análisis de las intervenciones de manera preventiva primaria o secundaria del deterioro cognitivo y/o demencias. La totalidad son intervenciones realizadas en otros países, en sujetos mayores de 60 años, en menor medida en sujetos institucionalizados y mayormente en personas mayores no institucionalizadas 
que se encuentran insertos en alguna comunidad, siendo seleccionados de manera aleatoria.

La selección de los estudios revisados permitió conocer intervenciones que provocan un impacto en el nivel cognitivo de las personas mayores, influyendo también en otras variantes de la vida tales como la funcionalidad, percepción de felicidad y sentimiento de sole ${ }^{-}$ dad.

En la totalidad de los estudios los programas aplicados a la población objetivo, influyeron de manera positiva en las escalas aplicadas previamente para medir el nivel cognitivo; tanto en personas con envejecimiento normal o con quejas de memoria o en sujetos en etapas ini ciales de demencias, mayormente en demencia tipo Alzheimer, retrasando en éstos el deterioro.

El proceso de selección de la evidencia permitió una validación interna, evitando errores sistemáticos o sesgos.

La evidencia revisada constituye una referencia técnica sobre las acciones que podrían aportar a las intervenciones locales para ser implementadas como programa a nivel local o política pública de salud, permitiendo disminuir u optimizar los gastos de tratamiento de las demencias, lo cual, según lo expues ${ }^{-}$ to representan un alto costo social, familiar y de recursos en salud. Otras varia- bles observadas en los estudios, tales como el nivel educacional, tiempo de lectura, nivel socioeconómico, sentimiento frente a la vida y condiciones físicas, son asociadas con los resultados principales en el rendimiento cognitivo.

En esta revisión no se declaran conflictos de intereses

\section{CONCLUSIÓN}

Según lo estudiado en la presente revisión sistemática, se pude concluir que como en toda patología existen factores de riesgos y factores protectores en la población, al conocer estos factores es posible estructurar intervenciones de promoción y prevención primaria y secundaria, tanto a nivel local como nacional, con el fin de potenciar los factores protectores y trabajar sobre los factores de riesgos modificables que presente cada individuo.

Es necesario promover, difundir y sensibilizar a la población sobre el tema, así como, estimular a los profesionales de salud a la investigación, desde las autoridades brindando herramientas, recursos y tiempos necesarios para su desarrollo e incorporando a nuevos actores sociales y al intersector. Esto también ayudaría a futuras revisiones con evidencia nacional, permitiendo así la implementación de programas efectivos 
en la población chilena o la mejora de los que ya se encuentren instaurados.

Los factores protectores y de riesgo para la presentación de deterioro cognitivo leve y demencias son similares a otras enfermedades crónicas no transmisibles; respondiendo a la pregunta de investigación, la evidencia encontrada muestra que el trabajo sobre factores protectores, tales como la actividad física, estimulación cognitiva, participación social, entre otros, permite mejorar el rendimiento cognitivo y retardar el avance de las demencias en etapas iniciales. Se requieren estudios locales y a largo plazo para observar la realidad nacional.

Sin duda el envejecimiento poblacional en Chile representa un desafío profesional e institucional, adaptando o transformando las intervenciones, haciendo necesario de esto un cambio, centrando las intervenciones hacia las comunidades y grupos organizados, con el apoyo y trabajo en conjunto con otros actores sociales, favoreciendo a ambas institucionalidades y lo que es más importante en la calidad de vida de los personas mayores, potenciando sus capacidades, viéndolos como sujetos de sabiduría representando un gran aporte hacia el desarrollo del país. 


\section{BiBLIOGRAFÍA}

1. Organización mundial de la Salud. [Internet].Ginebra, Suiza: Organización Mundial de la Salud [Citado el 9 de julio 2017]. Disponible desde: http://apps.who.int/iris/bitstream/ 10665/186466/1/9789240694873_spa.pdf

2. Ministerio de Salud, Gobierno de Chile. [Internet].Santiago, Chile: Ministerio de Salud [Citado el 9 de julio 2017]. Disponible desde: http://web.minsal.cl/sites/default/files/files/Borrador\%20documento $\% 20$ Programa $\% 20$ Nacional\%20 de $\% 20 \mathrm{Perso}^{-}$ nas\%20Adultas\%20Mayores-\%2004-03_14.pdf.

3. Matías-Guiu A, Pérez-Martínez A, Matías-Guiu J. A pilot study of a new method of cognitive stimulation using abacus arithmetic in healthy and cognitively impaired elderly subjects. . [Internet]. 2016, Jun. [Citado el 16 de julio 2017].; 31(5), 326331. Disponible desde: https://www.ncbi.nlm.nih.gov/pubmed/25840607

4. Gracia A, Santabárbara J, López R., Tomás C, Marcos G. Occupation and Risk of Cognitive Impairment and Dementia in People in over 55 Years: A Systematic Review. . [Internet]. 2016, Jun [Citado el 17 de julio 2017].; 90. Disponible desde: https://www.ncbi.nlm.nih.gov/pubmed/27325121

5. Rey Cao A, Canales I. Mejoras cognitivas y perceptivo-motrices en personas mayores participantes en un programa de estimulación integral cognitivo-motriz. Rev. bras. geriatr. gerontol. [Internet]. 2012, Ene. [Citado el 6 de Ago del 2017]; 15(1): 27-39. Disponible desde: http://dx.doi.org/10.1590/ S1809-98232012000100004.

6. Carballo-García V, Arroyo-Arroyo M, PorteroDíaz M, De León J. Effects of non-pharmacological therapy on normal ageing and on cognitive decline: Reflections on treatment objectives. Neurología (English Edition) [Internet]. 2012, Ago. [Citado el 6 de Ago del 2017]; 28(3), 160-168. Disponible desde: https://www.ucm.es/data/cont/docs/ $\begin{array}{lllllllllllllll} & 1 & 4 & 0 & - & 2 & 0 & 1 & 4 & - & 0 & 2 & - & 1 & 8\end{array}$ Publicacion_26\%20Art\%C3\%ADculoTNFDeterioroCognitivoNeurolog\%C3\%ADa_2013.pdf

7. Esteve M, Gil Á. El hábito de lectura como factor protector de deterioro cognitivo. Gaceta Sanitaria [Internet].2012, Jun. [Citado el 6 de Ago del 2017]; 27(1). Disponible desde: http://scielo.isciii.es/pdf/ gs/v27n1/original_breve4.pdf

8.- Clta Buchman A, Boyle P, Yu L, Shah R, Wilson $R$, Bennett $D$. Total daily physical activity and the risk of $A D$ and cognitive decline in older adults. Neurology. [Internet].2012, Abr. [Citado el 6 de Ago del 2017]; 78(17):1323-9. Disponible desde: http:// pubmedcentralcanada.ca/pmcc/articles/PMC3335448/

9. Cheng S, Chow P, Song Y, Yu E, Chan A, Lee T, Lam J. Mental and physical activities delay cognitive decline in older persons with dementia. The
American journal of geriatric psychiatry : official journal of the American Association for Geriatric Psychiatry. [Internet].2014, Jun. [Citado el 6 de Ago del 2017];22 (1). Disponible desde: https:// www.ncbi.nlm.nih.gov/pubmed/23582750

10. Maki Y, Ura C, Yamaguchi T, Murai T, Isahai $M$, Kaiho A, Yamagami T, Tanaka S, Miyamae F, Sugiyama M, Awata S, Takahashi R, Yamaguchi $H$. Effects of intervention using a community-based walking program for prevention of mental decline: a randomized controlled trial. Journal of the American Geriatrics Society [Internet]. 2012, Mar. [Citado el 6 de Ago del 2017]; 60 (3). Disponible desde: https://www.ncbi.nlm.nih.gov/pubmed/22288578

11.- Wang $H$, Jin $Y$, Hendrie $H$, Liang $C$, Yang $L$, Cheng Y, Unverzagt F, Ma F, Hall K, Murrell J, Li P, Bian J, Pei J, Gao S. Late life leisure activities and risk of cognitive decline. The journals of gerontology. Series A, Biological sciences and medical sciences [Internet]. 2013, Feb. [Citado el 6 de Ago del 2017]; 68 (2). Disponible desde: https:// www.ncbi.nlm.nih.gov/pubmed/22879456

12. Lam L, Chau R, Wong B, Fung A, Tam C, Leung G, Kwok T, Leung T, Ng S, Chan W. A 1year randomized controlled trial comparing mind body exercise (Tai Chi) with stretching and toning exercise on cognitive function in older Chinese adults at risk of cognitive decline. Journal of the American Medical Directors Association [Internet]. 2012, Jul. [Citado el 6 de Ago del 2017]; 13 (6). Disponible desde: https://s3.amazonaws.com/academia.edu.documents $/ 46842229 /$ j.jamda. 2012.03.00820160627-31963-1sbzqcf.pdf?AWSAccessKeyld=AKIAIWOWYYGZ2Y53UL3A\&EXpires $=1502850615 \&$ Signature $=\% 2 \mathrm{FqGxTcgN}$ diasS\%2By9Ue4W\%2B89DNao\%3D\&response$\begin{array}{llllllllll} & 0 & n & t & e & n & t & -\end{array}$ disposition=inline\%3B\%20filename\%3DA_1Year_Randomized_Controlled_Trial_Com.pdf

13 Shankar A, Hamer M, McMunn A, Steptoe A. Social isolation and loneliness: relationships with cognitive function during 4 years of follow-up in the English Longitudinal Study of Ageing. Psychosomatic medicine [Internet]. 2013, Feb. [Citado el 6 de Ago del 2017]; 75 (2). Disponible desde: https:// www.ncbi.nlm.nih.gov/pubmed/23362501

14. Nagamatsu LS, Chan A, Davis JC, Beattie BL, Graf P, Voss MW, Sharma D, Liu-Ambrose T. Physical activity improves verbal and spatial memory in older adults with probable mild cognitive impairment: a 6-month randomized controlled trial. Journal of aging research [Internet]. 2013, Feb. [Citado el 6 de Ago del 2017]; 2013:861893. Disponible desde: https://www.ncbi.nlm.nih.gov/pubmed/ 23509628

15. Holwerda TJ, Deeg DJ, Beekman AT, van Tilburg TG, Stek ML, Jonker C, Schoevers RA. Feelings of loneliness, but not social isolation, predict dementia onset: results from the Amsterdam Study 
of the Elderly (AMSTEL). Journal of neurology, neurosurgery, and psychiatry [Internet]. 2014, Feb. [Citado el 6 de Ago del 2017]; 85 (2). Disponible desde: https://www.ncbi.nlm.nih.gov/pubmed/ 23232034

16. Suzuki T, Shimada H, Makizako H, Doi T, Yoshida $D$, Ito $K$, Shimokata $H$, Washimi $Y$, Endo $H$, Kato T. A randomized controlled trial of multicomponent exercise in older adults with mild cognitive impairment. PloS one. [Internet]. 2013, Abr. [Citado el 6 de Ago del 2017]; 8(4):e61483. Disponible desde: https://www.ncbi.nlm.nih.gov/pubmed/ 23585901

17. Matías-Guiu A, Pérez-Martínez A, Matías-Guiu J. A pilot study of a new stimulation using abacus arithmetic in healthy and cognitively impaired elderly subjects. . [Internet]. 2016, Jun. [Citado el 16 de julio 2017].; 31(5), 326331. Disponible desde: https://www.ncbi.nlm.nih.gov/pubmed/25840607

18. Leiva-Saldaña A, Sánchez-Ramos J, LeónJariego J,Palacios-Gómez L. Factores predictores de deterioro cognitivo en población mayor de 64 años institucionalizada y no institucionalizada. Enfermería Clínica [Internet].2016, Mar. [Citado el 6 de Ago del 2017]; 26(2), 129-136. Disponible desde: http://www.sciencedirect.com/science/article/pii/S1130862115001424 